\title{
Determination of the embedded electronic states at nanoscale interface via surface-sensitive photoemission spectroscopy
}

\author{
Hui-Qiong Wang ${ }^{1,2,3 凶}$, Jiayi X $\mathrm{u}^{3}$, Xiaoyuan $\mathrm{Lin}^{3}$, Yaping $\mathrm{Li}^{1,4}$, Junyong Kang ${ }^{1}$ and Jin-Cheng Zheng $\mathbb{B}^{1,2,3 凶}$
}

\begin{abstract}
The fabrication of small-scale electronics usually involves the integration of different functional materials. The electronic states at the nanoscale interface plays an important role in the device performance and the exotic interface physics. Photoemission spectroscopy is a powerful technique to probe electronic structures of valence band. However, this is a surface-sensitive technique that is usually considered not suitable for the probing of buried interface states, due to the limitation of electron-mean-free path. This article reviews several approaches that have been used to extend the surfacesensitive techniques to investigate the buried interface states, which include hard X-ray photoemission spectroscopy, resonant soft X-ray angle-resolved photoemission spectroscopy and thickness-dependent photoemission spectroscopy. Especially, a quantitative modeling method is introduced to extract the buried interface states based on the film thicknessdependent photoemission spectra obtained from an integrated experimental system equipped with in-situ growth and photoemission techniques. This quantitative modeling method shall be helpful to further understand the interfacial electronic states between functional materials and determine the interface layers.
\end{abstract}

\section{Introduction}

\section{Important roles of interfaces}

An interface in physical science or materials science is usually defined as the boundary between two different materials or different physical states of the same matter. Interfaces can be classified into several typical catalogs, such as solid-gas interface, solid-liquid interface, liquid-liquid interface, and solid-solid interface, and so on. Surface is a special interface, formed between material and vacuum. The interface is important and interesting because its properties and behavior can be quite different from the adjacent bulk phases. The emergence of the interesting phenomena is resulted

Correspondence: Hui-Qiong Wang (hqwang@xmu.edu.cn) or

Jin-Cheng Zheng (jczheng@xmu.edu.cn)

${ }^{1}$ Fujian Provincial Key Laboratory of Semiconductors and Applications,

Collaborative Innovation Center for Optoelectronic Semiconductors and

Efficient Devices, Department of Physics, Xiamen University, Xiamen 361005 .

China

2Department of Physics, Xiamen University Malaysia, Sepang 43900, Malaysia

Full list of author information is available at the end of the article from the thermodynamic constraints enforced by the twodimensional surface/interface. The different concentrations (or density) and structural arrangements of atoms or molecules in the surface/interface region compared with bulk materials, result in unique physical and chemical properties of interfaces. Interfaces, therefore, are often found to play a central role both in nature and within a variety of different technological applications, devices, and industrial processes ${ }^{1}$. For example, solid-gas interfaces have been involved in catalytic reactions such as the reduction of harmful gas emissions in catalytic converter in automobiles, producing industrial chemicals through heterogeneous catalytic reactions, thin-film growth during microelectronics processing, etc. Liquid-gas interfaces are important for environmental problems. Liquid-liquid interfaces play a large role in the biological process and many daily life applications including detergents, foods, and paints by the stabilization of emulsions and microemulsions. Solid-solid interfaces are especially important in advanced functional materials and devices ${ }^{1,2}$.

\section{(c) The Author(s) 2021}

(c) (i) Open Access This article is licensed under a Creative Commons Attribution 4.0 International License, which permits use, sharing, adaptation, distribution and reproduction cc) in any medium or format, as long as you give appropriate credit to the original author(s) and the source, provide a link to the Creative Commons license, and indicate if changes were made. The images or other third party material in this article are included in the article's Creative Commons license, unless indicated otherwise in a credit line to the material. If material is not included in the article's Creative Commons license and your intended use is not permitted by statutory regulation or exceeds the permitted use, you will need to obtain permission directly from the copyright holder. To view a copy of this license, visit http://creativecommons.org/licenses/by/4.0/. 
Table 1 List of surface and interface analyzing techniques ${ }^{19,20}$ according to their detection features

\begin{tabular}{llll}
\hline Photon detection methods & $\begin{array}{l}\text { Electron detection } \\
\text { methods }\end{array}$ & $\begin{array}{c}\text { Neutron detection } \\
\text { methods }\end{array}$ & Ion detection methods \\
\hline TXRF and standing wave XRF, & XPS, & Neutron reflectivity & SIMS, \\
Energy dispersive and wavelength-dispersive XRF, & AES, & Neutron diffraction and & Electron impact (EI)- \\
Glancing-incidence (GI)-X-ray reflectivity (XRR), & EELS, & SNMS, \\
Gl-X-ray diffuse scattering, & APS, & Laser-SNMS, \\
Gl-resonant X-ray scattering, Gl-XRD Gl-XAFS, OES, Laser ablationg & RBS, \\
Or sputter depth profiling, & SEM, & LEIS, \\
lon-beam spectrochemical analysis, & EF-TEM, & ERDA, \\
RAIRS, & STM, & NRA, \\
ATR, & LEED & FIM \\
SEIRA, & & & \\
ATR-FTIR, & & & \\
Surface Raman spectroscopy, & & \\
Optical reflectivity and ellipsometry, & & \\
SFG, & & & \\
SXAPS, & & & \\
IPES & & & \\
\hline
\end{tabular}

Please refer to Appendix 1 for the explanation of acronyms

The nanoscale interfacial properties between functional materials can significantly affect a wide range of device characteristics, especially for modern microelectronics. Such effect would either hinder the performance of electronics or actually open opportunities for innovative design of new type of devices. For example, transition metal oxides, which exhibit rich material properties due to the unique characteristics of their outer $d$ electrons, are promising for the next-generation oxide electronics ${ }^{2-5}$. Both atom reconstruction and electron reconstruction, as well as spin, orbital, and charge coupling at the oxide interfaces have led to novel interface physics as well as emergent phenomena ${ }^{6-10}$. The conductivity, as well as superconductivity observed at the interface between the two wide-bandgap insulators of $\mathrm{LaAlO}_{3}$ and $\mathrm{SrTiO}_{3}$, are remarkable examples ${ }^{8,11-15}$. Heterojunctions that hybrid with semiconductors have also demonstrated significant roles in photocatalysts ${ }^{16-18}$. It is thus of great importance to characterize the electronic states at the interface.

\section{Characterization of interfaces}

Because of the importance of interfaces, many different tools or methods have been developed to characterize the chemical composition, geometrical arrangements, and various properties, including mechanical properties and processes (such as thickness, roughness, clusters/particles dimensions, and distribution, friction, fracture, strength, strain, stress, deformation properties, fatigue resistance, wear, etc.), physical properties and processes (e.g., density, crystallization, physical inter-diffusion, dielectric and magnetic properties, energy density, etc.), chemical properties and chemical processes (e.g., elemental and molecular compositions of the layers, size, and orientation of individual molecules, adhesion, corrosion, passivation, interfacial interactions, chemical diffusion, barrier properties, etc.), as well as optical properties and processes (including refractive indices, spectral reflectivity, and transmittance, optical absorption properties, etc. $)^{19}$. The characterization methods can be classified into different groups regarding the properties of objects studied, or detection features of tools. The classifications of the surface and interface analyzing techniques are available in several reviews and books ${ }^{19-23}$. Following the classification presented in refs. ${ }^{19,20}$, we listed the main surface and interface analysis techniques in Table 1 , according to the detection features. Typical analytical methods for buried interfaces include electron detection methods, photon detection methods, neutron detection methods, ion detection methods, and scanning probe methods.

\section{Typical analytical methods for buried interfaces}

Emerging electron microscopy techniques, based on scanning transmission electron microscopy (STEM) and/ or electron energy loss spectroscopy (EELS) (such as 4DSTEM, cryo-STEM, and monochromated EELS) are very useful tools for probing functional interfaces in energy materials (as shown typically in Fig. 1) ${ }^{24,25}$. Spatially resolved EELS is capable of examining the conduction 


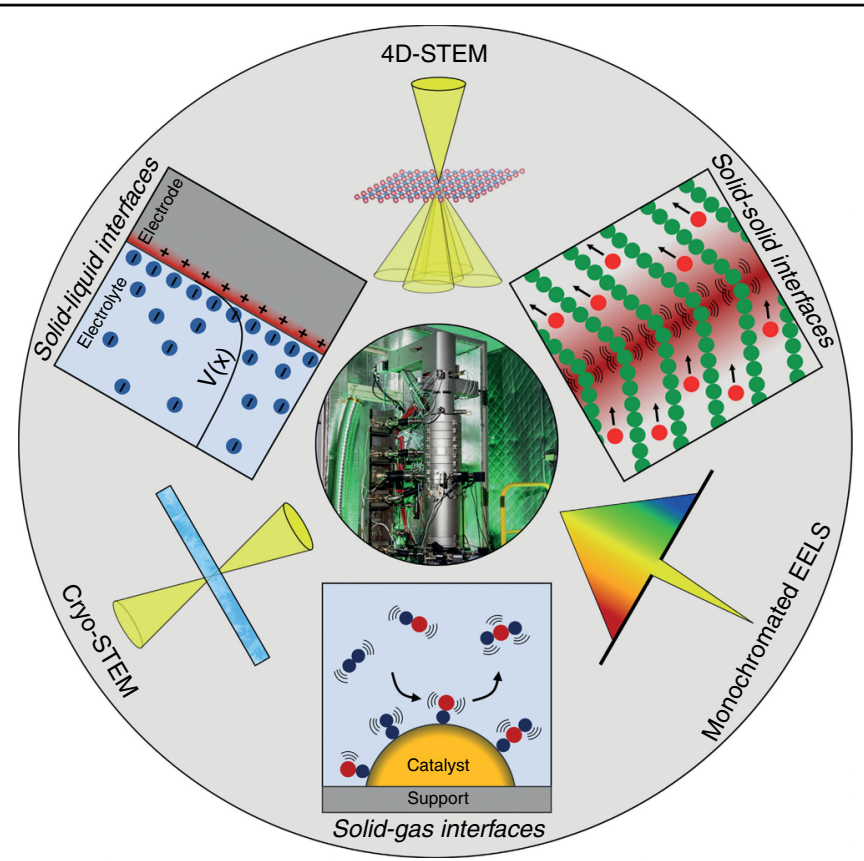

Fig. 1 Overview of probing functional interfaces in energy materials using emerging electron microscopy techniques. Reproduced with permission ${ }^{24}$. Copyright 2019, Wiley

band structure and has been used to study the electronic changes at perovskite oxide heterointerfaces ${ }^{7,26,27}$. However, EELS is usually equipped with the expensive facility of STEM and is also limited by the time-consuming, destructive sample preparation necessary for generating electron transparent specimens. Cathodoluminescence (CL) is capable of probing the emission properties at the interface area ${ }^{28,29}$. Photoemission spectroscopy (PES), including X-ray photoemission spectroscopy (XPS) and Ultraviolet photoemission spectroscopy (UPS) are powerful to investigate the valence band structure while X-ray absorption spectra (XAS) is frequently used for conduction band investigation. However, they are usually classified as "surface sensitive techniques", due to the limitation of electron mean free path. This review aims to introduce the development and extension of these techniques to probe the buried electronic states at the interface.

\section{Determination of interface electronic states using photoemission spectroscopy}

In general, there are three approaches to extend the application of "surface sensitive" photoemission spectroscopy to study buried interfaces: (1) Tuning the photon energy; (2) Adjusting the probing angle with respect to the surface normal; (3) Capturing thickness-dependent photoemission spectra. In this section, we will review the use of hard X-ray photoemission and resonant soft X-ray angle-resolved photoemission for probing interface electronic states, which increase the profiling depth by increasing the photon energy. A quantitative modeling method will then be introduced to extract the buried interface electronic states based on thickness-dependent photoemission spectra.

\section{Hard X-ray photoemission spectroscopy}

While the photon energies of X-rays used in the regular research laboratories for photoemission spectroscopy are usually limited to $1486.7 \mathrm{eV}$ ( $\mathrm{Al} \mathrm{K \alpha}$ radiation) or $1253.6 \mathrm{eV}$ ( $\mathrm{Mg} \mathrm{K \alpha}$ radiation), the development of synchrotron radiations has made it possible to tune the photon energies in a wide spectral range from infrared to hard X-rays ${ }^{30}$. The maximal probing depth is defined as $3 \lambda \cos \theta$, where $\lambda$ is the effective inelastic mean free path (IMFP), and $\theta$ is the angle between the detection direction and the surface normal (as shown in Fig. 2a) ${ }^{31}$. The mean free path of photoelectrons escaping from the solid as a function of kinetic energy ${ }^{32,33}$ is shown in Fig. $2 b$.

The angle-dependent XPS as a nondestructive method was often used for the characterization of chemical composition and electronic structure of ultrathin layers such as tin oxide films ${ }^{34}$, heterostructures between functional oxides ${ }^{35}$ or semiconductors ${ }^{36-38}$. The angledependent hard $\mathrm{x}$-ray photoemission spectroscopy (with $h v=3 \mathrm{keV}$ ) has been performed at Berlin Electron Storage Ring Society for Synchrotron Radiation to analyze the depth-profiled interface electron gas of $\mathrm{LaAlO}_{3} /$ $\mathrm{SrTiO}_{3}$ heterostructures, and the results supported an electronic reconstruction in the $\mathrm{LaAlO}_{3}$ overlayer as the 

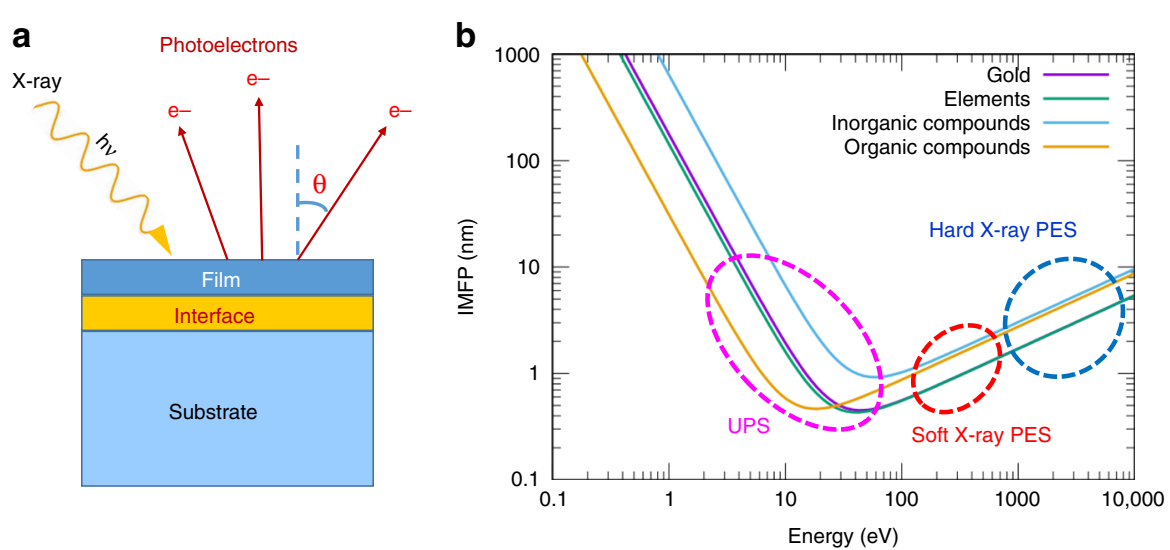

Fig. 2 Probing interface by adjusting the probing angle or tuning the photo energy. a Depth profiling by angle-resolved X-ray photoemission spectroscopy. $\mathbf{b}$ The inelastic mean free path (IMFP, $\lambda$ ) of photoelectron with different kinetic energy ranges indicated. (The IMFP plots are generated using formulas 5 and parameters 1 reported by Seah and Dench" ${ }^{32}$, namely, $\lambda=\frac{177}{E^{2}}+0.054 \sqrt{E}$ for "Gold", $\lambda=\frac{143}{E^{2}}+0.054 \sqrt{E}$ for "Elements",

$\lambda=\frac{641}{E^{2}}+0.096 \sqrt{E}$ for "Inorganic Compounds", and $\lambda=\frac{31}{E^{2}}+0.087 \sqrt{E}$ for "Organic Compounds". The units of $\lambda$ and $E$ are nm and eV, respectively.)

driving force for the 2D electron gas (2DEG) formation ${ }^{35}$. Using hard XPS, the impact of oxygen on the band structure at the $\mathrm{Ni} / \mathrm{GaN}$ interface was revealed ${ }^{36}$, the band edge profiles at the semiconductor heterostructures were extracted $^{37}$, and the core-level shifts at the buried $\mathrm{GaP} / \mathrm{Si}$ (001) interfaces were reported ${ }^{38}$. Aforementioned typical studied cases already demonstrated the powerful capability of hard XPS for buried interface analysis. A recent study further showed that the detection of deeply buried layers beyond the elastic limit can be enabled by inelastic background analysis, which demonstrated the potential for the characterization of deeply buried layers using synchrotron and laboratory-based hard XPS ${ }^{39}$.

\section{Resonant soft X-ray angle-resolved photoemission spectroscopy}

Although hard XPS is capable to provide valuable and even quantitative information on the electronic properties at interface, it still has some drawbacks. For example, the evidence of 2DEG at oxide heterointerface is indirect since the states at the Fermi level actually hosting the mobile electrons cannot be observed due to the small cross-sections of the photoabsorption at high photon energies, and thus the photoemission signals at the Fermi level are usually unfortunately missing. However, by exciting with photons tuned to an appropriate absorption edge, the resonant photoemission allows for a selective enhancement of the emission from orbitals with a given symmetry. Therefore, a combination of soft X-ray angleresolved photoemission spectroscopy (ARPES) with resonant photoexcitation can overcome this limitation, which has been demonstrated recently ${ }^{40-44}$.

The electronic structures of materials are characterized by three parameters of the electrons therein, namely, energy $(E)$, momentum $(k)$ and spin $(s)$. ARPES is often used to investigate the k-space electronic structures of buried interfaces. In a resonant ARPES experiment, polarization-controlled synchrotron radiation was used to map the electronic structure of buried conducting interfaces of $\mathrm{LaAlO}_{3} / \mathrm{SrTiO}_{3}{ }^{45}$. By combining X-ray photoelectron diffraction and ARPES, the interplay between electronic and structural properties in the $\mathrm{Pb} / \mathrm{Ag}(100)$ interface has been studied ${ }^{46}$. A critical thickness for the 2DEG formation in $\mathrm{SrTiO}_{3}$ embedded in $\mathrm{GdTiO}_{3}$ was observed by resonant ARPES ${ }^{47}$. Electronic structure of a buried quantum dot system (In, Mn)As, grown by molecular beam epitaxy, was investigated by soft-X-ray ARPES, which combines its enhanced probing depth with elemental and chemical state specificity achieved with resonant photoexcitation ${ }^{48}$. Using the similar soft-X-ray ARPES technique, the electronic structure of the buried $\mathrm{EuO} / \mathrm{Si}$ interface with momentum resolution and chemical specificity was probed ${ }^{49}$. The electronic structure measurements of the buried $\mathrm{LaNiO}_{3}$ layers in (111)-oriented $\mathrm{LaNiO}_{3} / \mathrm{LaMnO}_{3}$ superlattices ${ }^{50}$, the buried $\mathrm{SiO}_{2} / \mathrm{SiC}$ interface $^{51}$, and the investigation of electronic phase separation at the $\mathrm{LaAlO}_{3} / \mathrm{SrTiO}_{3}$ interfaces ${ }^{52}$, were reported using soft X-ray ARPES. By combining ARPES with soft X-ray standing-wave excitation, Gray et al. ${ }^{53}$ provided a detailed study on the buried interface in a $\mathrm{La}_{0.7} \mathrm{Sr}_{0.3} \mathrm{MnO}_{3} / \mathrm{SrTiO}_{3}$ magnetic tunnel junction. A recent topical review on ARPES studies of low-dimensional metallic states confined at insulating oxide surface and interfaces was reported by Plumb and Radovic ${ }^{54}$.

\section{Thickness dependent photoemission spectra}

It is also a common approach to capture a series of photoemission and/or absorption spectra as a function of 


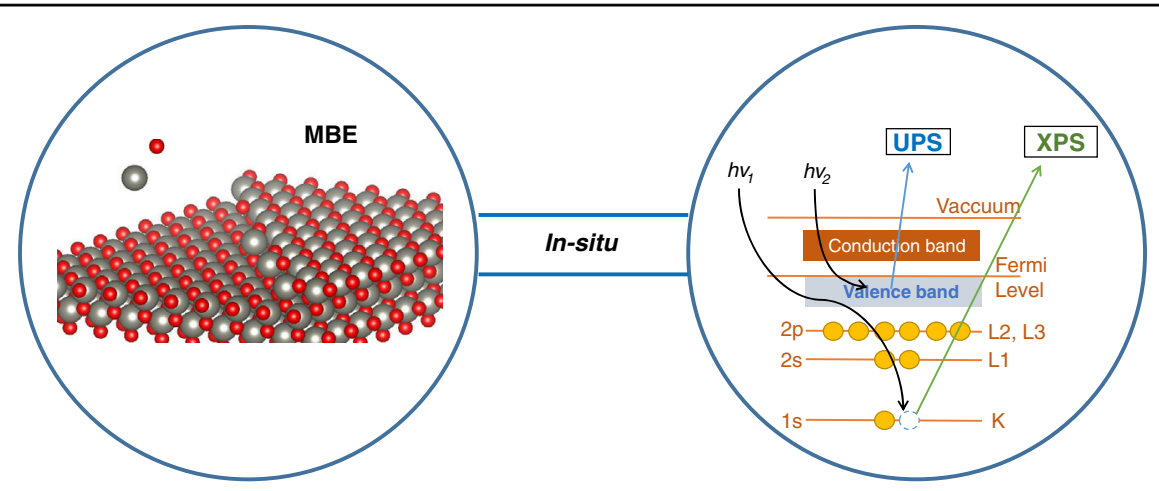

Fig. 3 Integrated ultra-high vacuum system for the quantitative modeling of photoemission spectra for interface states. Left: Thin film growth techniques (e.g., MBE). Right: In-situ photoemission spectroscopy (XPS and/or UPS)

film thickness and track the evolution of the spectrum features. Diebold et al. ${ }^{55}$ detected a crystalline ternary $\mathrm{MnTiO}_{x}$ at the interface of $\mathrm{Mn} / \mathrm{TiO}_{2}$ by the PES and XAS. The $\mathrm{Mn} / \mathrm{TiO}_{2}(110)$ was observed to consist of the reduced $\mathrm{Ti}$ cations and oxidized Mn overlayer atoms when deposited at $25^{\circ} \mathrm{C}$, while the interfacial Ti cations in the Mn films which were annealed to $\sim 650^{\circ} \mathrm{C}$ were found to be reoxidized to the $\mathrm{Ti}^{4+}$ state and the interfacial local order was enhanced at the same time. Gao et al. identified a $\mathrm{Fe} / \mathrm{Si}$ interfacial layer which was due to the chemisorption of the first Fe layer on the Si substrate, through the study of the thickness dependence of the Fe absorption signal on the substrate from in situ XAS measurements ${ }^{56}$. The electronic structure of the $\mathrm{TiO}_{2}-\mathrm{Al}_{2} \mathrm{O}_{3}$ interface was also investigated by the detailed analysis of the XAS Ti $2 p$ spectra as a function of the $\mathrm{TiO}_{2}$ deposition on $\mathrm{Al}_{2} \mathrm{O}_{3}{ }^{57}$, revealing the formation of $\mathrm{Ti}-\mathrm{O}-\mathrm{Al}$ cross-linking bonds at the interface, which could be attributed to the significant lowering of the crystal field of Ti atoms at the interface. A characterization technique based on the atomic core-level shifts was proposed by Holmstrom et al. ${ }^{58}$ to analyze the interfacial quality of the layered structures; high kineticenergy photoelectron spectroscopy with longer mean-free paths was also used to capture signals from the embedded interface layers. Gonzalez-Elipe and Yubero ${ }^{59}$ investigated chemical states and bonding configurations at the interfaces, mostly by probing the Auger parameter measured by $\mathrm{X}$-ray photoemission.

However, there was usually a lack of presentation of the spectra of interface states that were distinguished from and excluded the contributions from the overlayer and the substrate. In the following section, we will review a quantitative modeling method to retreat the interface state spectra and determine the interface layer structure, using UPS spectra. UPS is one of the probes that are sensitive to the electronic density-of-states near Fermi level $^{60}$, which are responsible to transfer charge along and across interfaces in device applications.

\section{Quantitative modeling of photoemission spectra for interface states}

\section{Requirement of experimental setup}

The quantitative probing method is to compare experimental PES spectra to model spectra as one material is grown on another. The experimental setup thus requires an integrated ultra-high vacuum (UHV) system that is equipped with the integration of thin-film growth techniques and spectra characterization probes, as shown in Fig. 3. A variety of thin film deposition techniques have been developed to meet the requirement of the high demand of different thinfilm materials, including molecular beam epitaxy $(\mathrm{MBE})^{61-66}$, pulsed laser deposition ${ }^{67-70}$, metal-organic chemical vapor deposition $^{71-73}$, atomic layer deposition ${ }^{74,75}$, etc. Oxide MBE has been particularly developed to fabricate novel oxide heterostructure and superlattice ${ }^{76}$. A typical oxide MBE chamber is usually equipped with different evaporation metal sources from either effusion cell or e-beam evaporator as well radio frequency plasma source to generate active oxygen atoms. Quartz crystal microbalance (QCM) would be adopted to determine the flux rate of the metal evaporation sources. In situ and real-time reflection high energy electron diffraction (RHEED) $)^{77-79}$ is chosen to precisely monitor the atomic layer growth as the pattern intensity oscillates simultaneously with the surface morphology during the growth. By comparing the RHEED patterns appearing at various zone axes, the symmetry of the surface structure can be determined; and by comparing those from the substrate and from the film, the registry relationship can be speculated. A differential pumping for the RHEED gun (electron source) is needed to prevent cathode filament degradation in the high partial oxygen pressure during oxide growth. During growth, the chamber is usually cooled by running water or liquid nitrogen.

By integrating the thin film growth system with spectroscopy characterization techniques connected under UHV channels, the fleshly grown thin films then have the privilege to undergo the characterization of electronic 


\section{a}

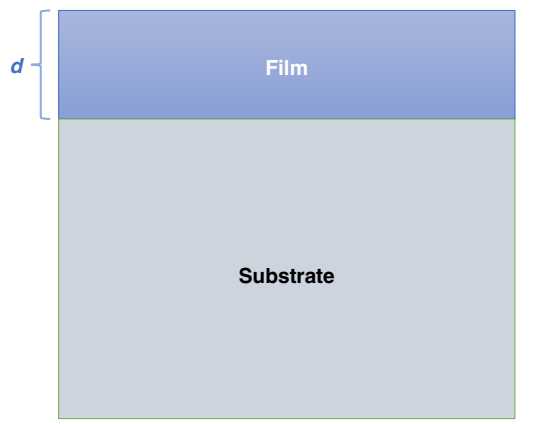

b

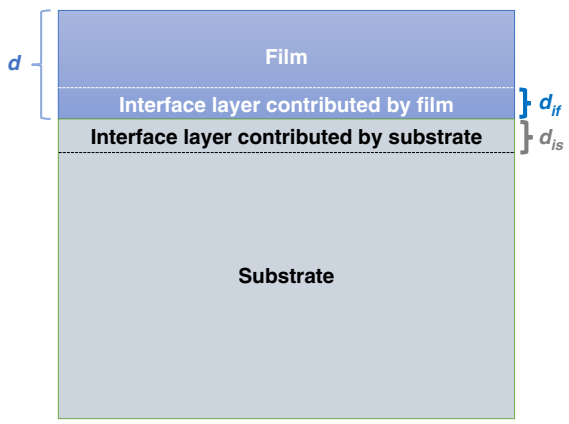

Fig. 4 The sketch models used for the quantitative simulation of spectra. a A film with a thickness of $d$ grown on the substrate, assuming no interface states. b. A film with a thickness of $d$ grown on the substrate, considering interface states. $d_{i f}$ and $d_{i s}$ are the thickness if the film and the substrate, respectively, involved to form the interface layer

properties without exposing to the air, thus keeping the natural features produced during growth. For the in situ characterization of XAS spectra that requires the tuning of photon energy, it would be necessary to attach a thin film growth chamber on the beam station of synchrotron radiation light source facility ${ }^{80,81}$. In regular laboratories, one can combine the thin film growth chamber with the analysis chamber that contains PES probing techniques ${ }^{82}$. For the PES experiment using the XPS instrument, X-rays with photon energy more than $1 \mathrm{kV}$ are generated by bombarding either magnesium or aluminum anodes with high-energy electrons. For the PES experiment using UPS equipment, ultraviolet photons are produced using a gas discharge lamp. Helium gas is usually used to emit photons with energies of $21.2 \mathrm{eV}$ ( $\mathrm{He} \mathrm{I}$ ) and 40.8 (He II). Recently, oxide MBE growth systems have been combined with angle-resolved photoemission to prompt the new research stage of strongly correlated materials ${ }^{83-90}$.

\section{Quantitative modeling of experimental spectra}

Even though PES spectra are considered surface sensitive, the mean-free path, $\lambda$, of the photoelectrons is large enough that the spectra will sample several monolayers into the sample. For thin films, the measured spectra will then consist of a superposition of emission from the substrate, from any interfacial states that may be present, and from the film, with each weighted by electron escape depths. The detailed analysis procedures are listed as follows.

\section{Step 1: (Assuming no interface states)}

Before examining any possible interface states, we first assume that there are no interface states present. We then compare the measured spectra to the model spectra consisting of a superposition of spectra from the substrate and that from the film. Assuming layer-by-layer growth, and taking into account the electron escape probability $e^{-\frac{d}{\lambda} 91}$, the spectral intensity $I$ as a function of thin-film thickness $d$ (as shown in Fig. 4a) can be calculated as ${ }^{92-94}$

$$
I_{\text {without interface }}^{\text {model }}(d)=I_{0}^{\text {Substrate }} e^{-\frac{d}{\lambda}}+I_{0}^{\text {Film }}\left(1-e^{-\frac{d}{\lambda}}\right)
$$

$I_{0}^{\text {Substrate }}$ and $I_{0}^{\text {Film }}$ represent the experimental intensity of the "bulk spectra" of the substrate and the film, respectively. In the previous reports ${ }^{92-94}$, the spectral intensity of the thickest film (usually not thinner than 20 monolayers) was used as the "bulk" spectrum. Here, we further take into account the thickness $D$ of the thickest film and $I_{D}^{\text {Film }} /\left(1-e^{-\frac{D}{\lambda}}\right)^{56}$ is adopted to represent the intensity of the "bulk" spectrum instead. Thus Eq. (1) is modified as

$$
I_{\text {without interface }}^{\text {model }}(d)=I_{0}^{\text {Substrate }} e^{-\frac{d}{\lambda}}+I_{D}^{\text {Film }}\left(1-e^{-\frac{d}{\lambda}}\right) /\left(1-e^{-\frac{D}{\lambda}}\right)
$$

The IMFP $\lambda$ in Eq. (2) can be estimated using the plots in Fig. $2 \mathrm{~b}$ or the formulas therein. It can also be calculated based on the reports by Tanuma et al. ${ }^{95,96}$. The thickness $d$ of the thinner film can be determined on the attenuation of the core-level photoemission line from the substrate ${ }^{97}$ :

$$
d=-\ln \frac{I_{\text {after }}}{I_{\text {before }}}
$$

where $I_{\text {before }}$ and $I_{\text {after }}$ are the spectral intensities of the XPS core-level photoemission line from the substrate before and after the thin film deposition with a thickness $d$. The thickness $D$ of the thickest film can be probed using a variety of techniques, including microscope.

Difference spectra are then taken between the experimental $I^{\text {expt }}$ and model spectra $I_{\text {without interface }}^{\text {model }}$

$$
\Delta I(d)=I^{\text {expt }}(d)-I_{\text {without interface }}^{\text {model }}(d)
$$


If there are no obvious features in the difference spectra $\Delta I(d)$, an electronically sharp interface without additional electronic state could be claimed.

\section{Step 2: (Considering interface states)}

Any difference features between the measured and model spectra may then result from the interfacial electronic structure. If an interface state exists, Eq. (2) can be changed to be: [modified from refs. ${ }^{92,93}$

$$
\begin{aligned}
I_{\text {with interface }}^{\text {model }}(d)= & I_{0}^{\text {Substrate }} e^{\frac{-\left(d+d_{i s}\right)}{\lambda}}+I_{D}^{\text {Film } \frac{1-e^{\frac{-\left(d-d_{i f}\right)}{\lambda}}}{1-e^{\frac{-D}{\lambda}}}} \\
& +I_{0}^{\text {Interface }}\left[1-e^{\frac{-\left(d_{i s}+d_{i f}\right)}{\lambda}}\right] e^{\frac{-\left(d-d_{i f}\right)}{\lambda}}
\end{aligned}
$$

where $d_{i s}$ and $d_{i f}$ are the thickness of the substrate and the deposited film, respectively, involved to form the interface layer (as shown in Fig. 4b); $I_{O}^{\text {Interface }}$ is the spectral intensity for the interface layer, assuming a semi-infinite slab having the interface electronic structure; and $d$ is the deposited thickness of the film. With the assumption that the experimental spectra contain interface states, we can use $I^{\text {expt }}$ for $I_{\text {with interface }}^{\text {model }}$ in Eq. (5). Therefore, Eq. (5) becomes

$$
\begin{aligned}
I^{\text {expt }}(d)= & I_{0}^{\text {Substrate }} e^{\frac{-\left(d+d_{i s}\right)}{\lambda}}+I_{D}^{F i l m} \frac{1-e^{\frac{-\left(d-d_{i f}\right)}{\lambda}}}{1-e^{\frac{-D}{\lambda}}} \\
& +I_{0}^{\text {Interface }}\left[1-e^{\frac{-\left(d_{i s}+d_{i f}\right)}{\lambda}}\right] e^{\frac{-\left(d-d_{i f}\right)}{\lambda}}
\end{aligned}
$$

Thus, the intensity of the interface state spectrum can be determined as

$$
I_{0}^{\text {Interface }}=\frac{I^{\text {expt }}(d)-\left\{I_{0}^{\text {Substrate }} e^{\frac{-\left(d+d_{i s}\right)}{\lambda}}+I_{D}^{F i l m} \frac{\left[1-e^{\frac{-\left(d-d_{i f}\right)}{\lambda}}\right]}{1-e^{\frac{-D}{\lambda}}}\right\}}{\left[1-e^{\frac{-\left(d_{i s}+d_{i f}\right)}{\lambda}}\right] e^{\frac{-\left(d-d_{i f}\right)}{\lambda}}}
$$

\section{Step 3: (Calculating interface states using different film thickness)}

Once the parameters $\lambda$, and film thickness $(d, D)$ are determined, the only variable parameters in Eq. (7) are $d_{\text {is }}$ and $d_{i f}$, which are the components of the interface layer thickness contributed by the substrate and the film, respectively. For certain interface model structure $d_{i s}$ and $d_{i f}$ (Fig. 4), one can calculate a set of $I_{0}^{\text {Interface }}$ data $\left(I_{0\left[d_{1}\right]}^{\text {Interface }}\right.$, $\left.I_{0\left[d_{2}\right]}^{\text {Interface }}, I_{0\left[d_{3}\right]}^{\text {Interface }}, \ldots\right)$ using the available experimental data
$I^{\text {expt }}(d)$ at different $d\left(d_{1}, d_{2}, d_{3} \ldots\right)$, based on Eq. (7). Different valuables of $d_{i s}$ and $d_{i f}$ for interface layer thickness can be used to obtain different $I_{0}^{\text {Interface }}$ sets of data. The most likely interface layer structure $d_{i s}$ and $d_{i f}$ would correspond to the particular $I_{0}^{\text {Interface }}$ set of data, in which case, $I_{0\left[d_{1}\right]}^{\text {Interface }}, I_{0\left[d_{2}\right]}^{\text {Interface }}, I_{0\left[d_{3}\right]}^{\text {Interface }} \ldots$ are similar to each other.

\section{Step 4: (Determining interface state spectrum based on the best-fit interface layer model)}

Once the best-fit interface layer model is determined, the interface state spectrum can be finally calculated by averaging the $I_{0}^{\text {Interface }}$ set of data with the corresponding values of the $d_{i s}$ and $d_{i f}$ parameters for the best-fit model.

\section{Case studies of the quantitative modeling of spectra for interface states}

The above-mentioned quantitative modeling has been used to study the interfaces between $\mathrm{Fe}_{3} \mathrm{O}_{4}$ and other transition-metal oxides, specifically $\mathrm{NiO}$ and $\mathrm{CoO}$. All of these oxides are of significant interest in spintronics. In particular, $\mathrm{Fe}_{3} \mathrm{O}_{4}-\mathrm{NiO}$ and $\mathrm{Fe}_{3} \mathrm{O}_{4}-\mathrm{CoO}$ have been proposed as an ingredient in all-oxide tunneling spin valves ${ }^{98}$. $\mathrm{Fe}_{3} \mathrm{O}_{4}$ is a metallic ferrimagnet, and both $\mathrm{NiO}$ and $\mathrm{CoO}$ are insulating antiferromagnets. The exchange biasing effect $^{99-103}$ in which the hysteresis loop of a ferro- or ferrimagnet is shifted asymmetrically along the field axis when in contact with an antiferromagnetic material, has been observed for both interfaces, making them interesting for spintronics. $\mathrm{NiO}$ and $\mathrm{CoO}$ have the same rocksalt crystal structure, and, although $\mathrm{Fe}_{3} \mathrm{O}_{4}$ has the inverse spinel structure, both structures share a common face-centered-cubic oxygen sublattice, where the lattice mismatch is only $0.55 \%$ between $\mathrm{Fe}_{3} \mathrm{O}_{4}$ and $\mathrm{NiO}$ and $1.45 \%$ between $\mathrm{Fe}_{3} \mathrm{O}_{4}$ and $\mathrm{CoO}$. Despite the fact that $\mathrm{NiO}$ and $\mathrm{CoO}$ have very similar bulk electronic properties, it is interesting that, the $\mathrm{Fe}_{3} \mathrm{O}_{4}(001)-\mathrm{NiO}$ (001) interface exhibits a sharp interface without obvious interface electronic state ${ }^{88}$, while the $\mathrm{Fe}_{3} \mathrm{O}_{4}(001)-\mathrm{CoO}(001)$ interface displays non-trivial electronic state and the interface state spectrum was determined using the above mentioned quantitative modeling by comparing two interface layer models $^{82}$. In one case, the interface layer consisted of one monolayer of the substrate $\mathrm{Fe}_{3} \mathrm{O}_{4}$ plus one monolayer of the film $\mathrm{CoO}$; in the other case, the interface layer consisted of only one monolayer of the film $\mathrm{CoO}$. The determination of the better-fit interface model was based on the observation of the degree of similarity among the generated three spectra of $I_{0\left[d_{1}\right]}^{\text {Interface }}, I_{0\left[d_{2}\right]}^{\text {Interface }}, I_{0\left[d_{3}\right]}^{\text {Interface }}$, using each model. It was concluded that the first case where the interface layer consists of one monolayer of the substrate $\mathrm{Fe}_{3} \mathrm{O}_{4}$ plus one monolayer of the film $\mathrm{CoO}$ is closer to the 


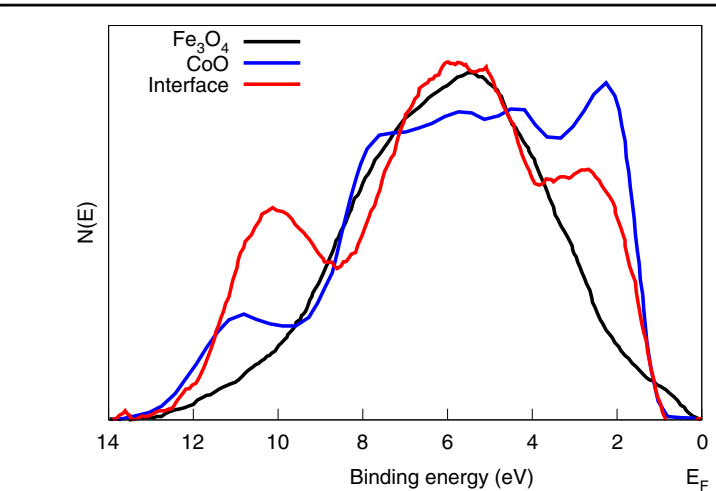

Fig. 5 The determined spectrum of interface states between $\mathrm{Fe}_{3} \mathrm{O}_{4}-\mathrm{CoO}$ based on the quantitative modeling method, compared with the substrate spectrum of $\mathrm{Fe}_{3} \mathrm{O}_{4}$ and the film spectrum of $\mathrm{CoO}$. Reproduced with permission (adapted from ${ }^{82}$ ). Copyright 2018, American Physical Society

actual case. The interface states spectrum determined using the best-fit model is shown in Fig. 5 .

\section{Discussions}

Although the quantitative modeling of experimental PES spectra as presented above is a simplified version to some extent, it captures the main feature of the interface states without losing the generality. More technical details or physics phenomenon can be included in the framework of aforementioned analysis procedure by modifying corresponding terms. One prospect related to PES technique is the photoelectron diffraction (PED) effects ${ }^{104-106}$. Due to the PED effects, the PES intensity can be modulated depending on the electron emission angles (polar and azimuthal) or energy of incident $x$-ray source ${ }^{104,105}$. For the angle-dependent PES, the formula can be further modified to include the effects of $\mathrm{x}$-ray incident angles and photoelectron collection angles on the emission intensity.

The other prospect is related to the structural and compositional nature of the interface region. For example, at oxide/oxide interfaces, cation mixing often occurs. In fact, our quantitative modeling procedure as presented above is valid for general electronic interface states, which is caused either by electronic reconstruction or by atomic re-arrangement including atom mixing at interface. Of course, in order to fully investigate the origin of the interface states, such as the composition and structure of the interface, one may combine quantitative spectra modeling with other characterization techniques such as synchrotron radiation XAS or scanning transmission electron microscopy (e.g., quantitative electron diffraction $^{107-109}$ or EELS, etc) or theoretical computations including first-principles calculations based on density functional theory to perform an integrated study ${ }^{109,110}$.

\section{Conclusion and future aspect}

In conclusion, this review article summaries several approaches that have been adopted to extend the application of surface-sensitive photoemission techniques to buried interfaces. A quantitative method is reviewed to extract the electronic states at the embedded interface between two functional materials, based on the thicknessdependent experimental photoemission spectra, which is one of the important techniques to determine the valance band the electronic structure at the interface. The quantitative model method also serves as an efficient and effective approach to determine the interface layer model involving the component layers from the substrate and the film, respectively.

It is expected that this quantitative modeling method could be extended to other electronic states probes and would have a broad application in probing interfacial electronic states, which are crucial for device performance. The current modeling method is based on the assumption that the film is deposited on the substrate surface following the ideal layer-by-layer growth mode. In the future, this modeling method can be further developed to take into account other possible growth modes.

\section{Acknowledgements \\ This work is supported by the National Natural Science Foundation of China (Grant No. U1332105) and by the Xiamen University Malaysia Research Fund (Grant no. XMUMRF/2019-C4/IORI/0002). \\ Author details \\ ${ }^{1}$ Fujian Provincial Key Laboratory of Semiconductors and Applications, Collaborative Innovation Center for Optoelectronic Semiconductors and Efficient Devices, Department of Physics, Xiamen University, Xiamen 361005, China. ${ }^{2}$ Department of Physics, Xiamen University Malaysia, Sepang 43900, Malaysia. ${ }^{3}$ Department of New Energy Science and Engineering, Xiamen University Malaysia, Sepang 43900, Malaysia. ${ }^{4}$ College of Science, Henan University of Technology, Zhengzhou 450001, China}

\section{Author contributions}

The manuscript was written through the contributions of all authors. All authors have given approval to the final version of the manuscript.

Competing interests

The authors declare no competing interests.

\section{Appendix 1. List of surface analysis acronyms ${ }^{20}$}

\section{Electron Excitation}

AES, Auger electron spectroscopy

BIS, Bremsstrahlung isochromat spectroscopy (or ILS, ionization loss spectroscopy)EDXS, Energy-dispersive X-ray spectroscopy

EELS, Electron energy loss spectroscopy

EFTEM, Energy-filtered transmission electron microscopy 
continued

ESD, Electron-stimulated desorption (or EID, electron-induced desorption)

ESDIAD, Electron-stimulated desorption ion angular distribution

IPES, Inverse photoemission spectroscopy

LEED, Low-energy electron diffraction

RHEED, Reflection high-energy electron diffraction

SXAPS, Soft X-ray appearance potential spectroscopy (or APS, appearance potential spectroscopy)

SAM, Scanning Auger microscopy

SEM, Scanning Electron microscopyEF-TEM, Energy-filtering transmission electron microscopy

2. Ion Excitation

ERDA, Elastic recoil detection analysis

GDMS, Glow discharge (GD) mass spectrometry

GD-OES, Glow discharge (GD) optical emission spectroscopy (OES)

IAES, Ion (excited) Auger electron spectroscopy

IBSCA, Ion beam spectrochemical analysis (or SCANIIR, surface composition by analysis of neutral and ion impact radiation or BLE, bombardment induced light emission)

INS, Ion neutralization spectroscopy

LEIS, Low-energy ion scattering (or ISS, lon-scattering spectroscopy)

NRA, Nuclear reaction analysis

RBS, Rutherford back-scattering spectroscopy (or HEIS, high-energy ion scattering)

SIMS, Secondary-ion mass spectrometry

(SSIMS, static secondary-ion mass spectrometry)

(DSIMS, dynamic secondary-ion mass spectrometry)

SNMS, Secondary neutral mass spectrometry

3. Photon Excitation

ELL, Ellipsometry

LA, Laser ablation

LIBS, Laser-induced breakdown spectroscopy (or LIPS, Laser-induced plasma spectroscopy)

RAIRS, Reflection-absorption infrared spectroscopy (or IRRAS, infrared reflection-absorption spectroscopy, or IRAS, infrared absorption spectroscopy, or ERIRS, external reflection infrared spectroscopy)

ATR, Attenuated total reflection

FTIR, Fourier transform infred spectroscopy

SERS, Surface-enhanced Raman scattering

SFG, Sum frequency generation

SHG, (optical) Second-harmonic generation

SNOM, Scanning near-field optical microscopyTXRF, Total reflection X-ray fluorescence (XRF) analysis continued

UPS, Ultraviolet photoelectron spectroscopy

XPS, X-ray photoelectron spectroscopy (or ESCA, electron spectroscopy for chemical analysis)

$X R D, X$-ray diffraction

XAFS, X-ray absorption fine structure

4. Neutral Excitation

FABMS, Fast-atom bombardment mass spectrometry

5. Thermal Excitation

TDS, Thermal desorption spectroscopy

6. High-Field Excitation

AP, Atom probe

FIM, Field ion microscopy

IETS, Inelastic electron tunneling spectroscopy

STM, Scanning tunneling microscopy

STS, Scanning tunneling spectroscopy

7. Mechanical Force

AFM, Atomic force microscopy

Received: 14 December 2020 Revised: 24 June 2021 Accepted: 6 July 2021 Published online: 27 July 2021

\section{References}

1. Williams, C. T. \& Beattie, D. A. Probing buried interfaces with non-linear optical spectroscopy. Surf. Sci. 500, 545-576 (2002).

2. Mannhart, J. \& Schlom, D. G. Oxide interfaces-an opportunity for electronics. Science 327, 1607-1611 (2010).

3. Ngai, J. H., Walker, F. J. \& Ahn, C. H. Correlated oxide physics and electronics. Annu. Rev. Mater. Res. 44, 1-17 (2014).

4. Ngai, J. H. et al. Electrically coupling complex oxides to semiconductors: a route to novel material functionalities. J. Mater. Res. 32, 249-259 (2017).

5. Mathews, S. et al. Ferroelectric field effect transistor based on epitaxial perovskite heterostructures. Science 276, 238-240 (1997).

6. Jackeli, G. \& Khaliullin, G. Spin, orbital, and charge order at the interface between correlated oxides. Phys. Rev. Lett. 101, 216804 (2008).

7. Ohtomo, A. et al. Artificial charge-modulationin atomic-scale perovskite titanate superlattices. Nature 419, 378-380 (2002).

8. Ohtomo, A. \& Hwang, H. Y. A high-mobility electron gas at the $\mathrm{LaAlO}_{3} / \mathrm{SrTiO}_{3}$ heterointerface. Nature 427, 423-426 (2004).

9. Gozar, A. et al. High-temperature interface superconductivity between metallic and insulating copper oxides. Nature 455, 782-785 (2008).

10. Hwang, H. Y. et al. Emergent phenomena at oxide interfaces. Nat. Mater. 11, 103-113 (2012).

11. Reyren, N. et al. Superconducting interfaces between insulating oxides. Science 317, 1196-1199 (2007).

12. Reiner, J. W., Walker, F. J. \& Ahn, C. H. Atomically engineered oxide interfaces. Science 323, 1018-1019 (2009).

13. Schlom, D. G. \& Pfeiffer, L. N. Upward mobility rocks! Nat. Mater. 9, 881-883 (2010).

14. Siemons, W. et al. Origin of charge density at $\mathrm{LaAlO}_{3}$ on $\mathrm{SrTiO}_{3}$ heterointerfaces: possibility of intrinsic doping. Phys. Rev. Lett. 98, 196802 (2007). 
15. Liu, Z. Q. et al. Origin of the two-dimensional electron gas at $\mathrm{LaAlO}_{3} / \mathrm{SrTiO}_{3}$ interfaces: the role of oxygen vacancies and electronic reconstruction. Phys. Rev. X 3, 021010 (2013)

16. Kumari, P. et al. Nanoscale $2 \mathrm{D}$ semi-conductors-Impact of structural properties on light propagation depth and photocatalytic performance. Sep. Purif. Technol. 258, 118011 (2021).

17. Mayer, M. T. et al. Forming heterojunctions at the nanoscale for improved photoelectrochemical water splitting by semiconductor materials: case studies on hematite. Acc. Chem. Res. 46, 1558-1566 (2013).

18. Gholipour, M. R. et al. Nanocomposite heterojunctions as sunlight-driven photocatalysts for hydrogen production from water splitting. Nanoscale $\mathbf{7}$ 8187-8208 (2015).

19. Stoev, K. \& Sakurai, K. Recent progresses in nanometer scale analysis of buried layers and interfaces in thin films by X-rays and Neutrons. Anal. Sci. 36 901-922 (2020).

20. Friedbacher, G. \& Bubert, H. Surface and Thin Film Analysis: A Compendium of Principles, Instrumentation, and Applications. 2nd edn. (Weinheim: Wiley$\mathrm{VCH}, 2011)$.

21. Imae, T. Nanolayer Research: Methodology and Technology for Green Chemistry. (Amsterdam: Elsevier, 2017).

22. González-Cobos, J. \& de Lucas-Consuegra, A. A review of surface analysis techniques for the investigation of the phenomenon of electrochemical promotion of catalysis with alkaline ionic conductors. Catalysts 6, 15 (2016).

23. Seah M., Chiffre L. Surface and Interface Characterization. In: Springer Handbook of Materials Measurement Methods. (eds Czichos H., Saito T., Smith L.) (Berlin: Springer 2006).

24. Zachman, M. J. et al. Emerging electron microscopy techniques for probing functional interfaces in energy materials. Angew. Chem. Int. Ed. 59, 1384-1396 (2020)

25. Zhou, $\mathrm{H}$. et al. Interfaces between hexagonal and cubic oxides and their structure alternatives. Nat. Commun. 8, 1474 (2017).

26. Nakagawa, N., Hwang, H. Y. \& Muller, D. A. Why some interfaces cannot be sharp. Nat. Mater. 5, 204-209 (2006)

27. Muller, D. A. et al. Atomic-scale chemical imaging of composition and bonding by aberration-corrected microscopy. Science 319, 1073-1076 (2008).

28. Brillson, L. J. Applications of depth-resolved cathodoluminescence spectroscopy. J. Phys. D: Appl. Phys. 45, 183001 (2012).

29. Chen, L. et al. Reversing abnormal hole localization in high-Al-content AIGaN quantum well to enhance deep ultraviolet emission by regulating the orbital state coupling. Light:: Sci. Appl. 9, 104 (2020).

30. Balerna, A. \& Mobilio, S. Introduction to synchrotron radiation. In: Synchrotron Radiation (ed Mobilio, S., Boscherini, F. \& Meneghini, C.) (Berlin: Springer, 2015).

31. Pryds, N. \& Esposito, V. Metal Oxide-Based Thin Film Structures. (Amsterdam: Elsevier, 2018).

32. Seah, M. P. \& Dench, W. A. Quantitative electron spectroscopy of surfaces: a standard database for electron inelastic mean free paths in solids. Surf. Interface Anal. 1, 2-11 (1979).

33. Cancellieri, C. \& Strocov, V. N. Spectroscopy of Complex Oxide Interfaces: Photoemission and Related Spectroscopies. (Cham: Springer, 2018).

34. Krzywiecki, M., Sarfraz, A. \& Erbe, A. Towards monomaterial p-n junctions: Single-step fabrication of tin oxide films and their non-destructive characterisation by angle-dependent $\mathrm{X}$-ray photoelectron spectroscopy. Appl. Phys. Lett. 107, 231601 (2015).

35. Sing, M. et al. Profiling the interface electron gas of $\mathrm{LaAlO}_{3} / \mathrm{SrTiO}_{3}$ heterostructures with hard x-ray photoelectron spectroscopy. Phys. Rev. Lett. 102 176805 (2009).

36. Mizushima, H. et al. Impact of oxygen on band structure at the Ni/GaN interface revealed by hard X-ray photoelectron spectroscopy. Appl. Phys. Lett. 118, 121603 (2021).

37. Sushko, P. V. \& Chambers, S. A. Extracting band edge profiles at semiconductor heterostructures from hard-x-ray core-level photoelectron spectra. Sci. Rep. 10, 13028 (2020)

38. Romanyuk, O. et al. Hard X-ray photoelectron spectroscopy study of core level shifts at buried GaP/Si(001) interfaces. Surf. Interface Anal. 52, 933-938 (2020).

39. Spencer, B. F. et al. Inelastic background modelling applied to hard X-ray photoelectron spectroscopy of deeply buried layers: a comparison of synchrotron and lab-based (9.25 keV) measurements. Appl. Surf. Sci. 541, 148635 (2021)
40. Kobayashi, M. et al. Digging up bulk band dispersion buried under a passivation layer. Appl. Phys. Lett. 101, 242103 (2012)

41. Drera, $\mathrm{G}$. et al. Spectroscopic evidence of in-gap states at the $\mathrm{SrTiO}_{3} / \mathrm{LaAlO}_{3}$ ultrathin interfaces. Appl. Phys. Lett. 98, 052907 (2011)

42. Koitzsch, A. et al. In-gap electronic structure of $\mathrm{LaAlO}_{3}-\mathrm{SrTiO}_{3}$ heterointerfaces investigated by soft x-ray spectroscopy. Phys. Rev. B 84, 245121 (2011).

43. Cancellieri, $\mathrm{C}$. et al. Interface Fermi states of $\mathrm{LaAlO}_{3} / \mathrm{SrTiO}_{3}$ and related heterostructures. Phys. Rev. Lett. 110, 137601 (2013).

44. Berner, G. et al. Direct k-space mapping of the electronic structure in an oxide-oxide interface. Phys. Rev. Lett. 110, 247601 (2013)

45. Cancellieri, $\mathrm{C}$. et al. Doping-dependent band structure of $\mathrm{LaAlO}_{3} / \mathrm{SrTiO}_{3}$ interfaces by soft $\mathrm{x}$-ray polarization-controlled resonant angle-resolved photoemission. Phys. Rev. B 89, 121412 (2014).

46. Crepaldi, A. et al. Interplay between electronic and structural properties in the Pb/Ag(1 0 0) interface. J. Phys.: Condens. Matter 27, 455502 (2015).

47. Nemšák, S. et al. Observation by resonant angle-resolved photoemission of a critical thickness for 2-dimensional electron gas formation in $\mathrm{SrTiO}_{3}$ embedded in $\mathrm{GdTiO}_{3}$. Appl. Phys. Lett. 107, 231602 (2015).

48. Bouravleuv, A. D. et al. Electronic structure of (In, Mn)As quantum dots buried in GaAs investigated by soft-x-ray ARPES. Nanotechnology 27, 425706 (2016)

49. Lev, L. L. et al. Band structure of the EuO/Si interface: justification for silicon spintronics. J. Mater. Chem. C. 5, 192-200 (2017).

50. Bruno, F. Y. et al. Electronic structure of buried $\mathrm{LaNiO}_{3}$ layers in (111)-oriented $\mathrm{LaNiO}_{3} / \mathrm{LaMnO}_{3}$ superlattices probed by soft x-ray ARPES. APL. Materials $\mathbf{5}$ 016101 (2017).

51. Woerle, J. et al. Electronic band structure of the buried $\mathrm{SiO}_{2} / \mathrm{SiC}$ interface investigated by soft x-ray ARPES. Appl. Phys. Lett. 110, 132101 (2017).

52. Strocov, V. N. et al. Electronic phase separation at $\mathrm{LaAlO}_{3} / \mathrm{SrTiO}_{3}$ interfaces tunable by oxygen deficiency. Physical Review. Materials 3, 106001 (2019).

53. Gray, A. X. et al. Momentum-resolved electronic structure at a buried interface from soft X-ray standing-wave angle-resolved photoemission. EPL 104 17004 (2013).

54. Plumb, N. C. \& Radovic, M. Angle-resolved photoemission spectroscopy studies of metallic surface and interface states of oxide insulators. J. Phys.: Condens. Matter 29, 433005 (2017).

55. Diebold, U. \& Shinn, N. D. Adsorption and thermal stability of $\mathrm{Mn}$ on $\mathrm{TiO}_{2}(110): 2 p$ X-ray absorption spectroscopy and soft X-ray photoemission. Surf. Sci. 343, 53-60 (1995)

56. Gao, X. Y. et al. Thickness dependence of X-ray absorption and photoemission in Fe thin films on Si (0 0 1). J. Electron Spectrosc. Relat. Phenom. 151 199-203 (2006).

57. Sánchez-Agudo, M. et al. Electronic interaction at the $\mathrm{TiO}_{2}-\mathrm{Al}_{2} \mathrm{O}_{3}$ interface as observed by X-ray absorption spectroscopy. Surf. Sci. 482-485, 470-475 (2001)

58. Holmström, E. et al. Sample preserving deep interface characterization technique. Phys. Rev. Lett. 97, 266106 (2006).

59. Nalwa, H. S. Handbook of Surfaces and Interfaces of Materials (San Diego: Academic Press, 2001)

60. Henrich, V. E. \& Cox, P. A. The Surface Science of Metal Oxides. (Cambridge: Cambridge University Press, 1994).

61. Chambers, S. A. Epitaxial growth and properties of thin film oxides. Surf. Sci. Rep. 39, 105-180 (2000).

62. Schlom, D. G. et al. A thin film approach to engineering functionality into oxides. J. Am. Ceram. Soc. 91, 2429-2454 (2008).

63. Franchi, S. Molecular beam epitaxy: fundamentals, historical background and future prospects. In: Molecular Beam Epitaxy: From Research to Mass Production (ed Henini, M.) (Amsterdam: Elsevier, 2013).

64. Schlom, D. G. \& Harris, J. S. Jr. MBE Growth Of High Tc superconductors. in Molecular Beam Epitaxy: Applications to Key Materials (ed Farrow, R. F. C.) (Park Ridge: Noyes, 1995), 505-622.

65. Bozovic, I. \& Schlom, D. G. Superconducting thin films: materials, preparation, and properties. In: Encyclopedia of Materials: Science and Technology (eds Buschow, K. H. J. et al.) (Amsterdam: Elsevier, 2001), 8955-8964.

66. Henini M. Molecular Beam Epitaxy: From Research to Mass Production (Amsterdam: Elsevier 2018)

67. Chrisey, D. B. \& Hubler, G. K. Pulsed Laser Deposition of Thin Films. (New York: Wiley, 1994).

68. Frey, T. et al. Effect of atomic oxygen on the initial growth mode in thin epitaxial cuprate films. Phys. Rev. B 49, 3483-3491 (1994). 
69. Koster, G. et al. Imposed layer-by-layer growth by pulsed laser interval deposition. Appl. Phys. Lett. 74, 3729-3731 (1999).

70. Brock, J. D. et al. Nucleation, coarsening, and coalescence during layer-bylayer growth of complex oxides via pulsed laser deposition: time-resolved, diffuse X-ray scattering studies. Mater. Sci. Eng.: A 528, 72-76 (2010).

71. de Keijser, M. \& Dormans, G. J. M. Chemical vapor deposition of electroceramic thin films. MRS Bull. 21, 37-43 (1996).

72. Roeder, J. F. et al. Liquid-delivery MOCVD: chemical and process perspectives on ferro-electric thin film growth. Adv. Mater. Opt. Electron. 10, 145-154 (2000).

73. Holloway, P. H. \& McGuire, G. E. Handbook of Compound Semiconductors: Growth, Processing, Characterization, and Devices. (Noyes, New Jersey1995).

74. Jaggernauth, A., Mendes, J. C. \& Silva, R. F. Atomic layer deposition of high-k layers on polycrystalline diamond for MOS devices: a review. J. Mater. Chem. C. 8, 13127-13153 (2020).

75. Maina, J. W. et al. Atomic layer deposition of transition metal films and nanostructures for electronic and catalytic applications. Crit. Rev. Solid State Mater. Sci. https://doi.org/10.1080/10408436.2020.1819200 (2020)

76. Schlom, D. G. Perspective: oxide molecular-beam epitaxy rocks. APL Mater. $\mathbf{3}$, 062403 (2015).

77. Ichimiya, A. \& Cohen, P. I. Reflection High-Energy Electron Diffraction. (Cambridge University Press, Cambridge, 2004).

78. Fisher, P. et al. Stoichiometric, nonstoichiometric, and locally nonstoichiometric $\mathrm{SrTiO}_{3}$ films grown by molecular beam epitaxy. J. Appl. Phys. 103, 013519 (2008).

79. Haeni, J. H., Theis, C. D. \& Schlom, D. G. RHEED intensity oscillations for the stoichiometric growth of $\mathrm{SrTiO}_{3}$ thin films by reactive molecular beam epitaxy. J. Electroceram. 4, 385-391 (2000).

80. Li, Y. P. et al. Interfacial electronic states of misfit heterostructure between hexagonal $\mathrm{ZnO}$ and cubic NiO. Phys. Rev. Mater. 4, 124601 (2020).

81. Oshima, M. et al. Combinatorial in situ Growth-and-analysis with synchrotron radiation of thin films for oxide electronics. AIP Conf. Proc. 879, 1667-1670 (2007).

82. Wang, H. Q., Altman, E. I. \& Henrich, V. E. Interfacial properties between CoO (100) and $\mathrm{Fe}_{3} \mathrm{O}_{4}$ (100). Phys. Rev. B 77, 085313 (2008).

83. $\mathrm{Xu}, \mathrm{H}$. C. et al. In situ engineering and characterization on the artificial heterostructures of correlated materials with integrated OMBE-ARPES. J. Electron Spectrosc. Relat. Phenom. 200, 347-355 (2015).

84. Chikamatsu, A. et al. Band structure and Fermi surface of $\mathrm{La}_{0.6} \mathrm{Sr}_{0.4} \mathrm{MnO}_{3}$ thin films studied by in situ angle-resolved photoemission spectroscopy. Phys. Rev. B 73, 195105 (2006).

85. Wadati, $\mathrm{H}$. et al. In situ photoemission study of $\mathrm{Nd}_{1-x} \mathrm{SrXMnO}_{3}$ epitaxial thin films. Phys. Rev. B 79, 153106 (2009).

86. Tebano, A. et al. Preferential occupation of interface bands in $\mathrm{La}_{2 / 3} \mathrm{Sr}_{1 / 3} \mathrm{MnO}_{3}$ films as seen via angle-resolved photoemission. Phys. Rev. B 82, 214407 (2010).

87. Wadati, $\mathrm{H}$. et al. Strong localization of doped holes in $\mathrm{La}_{1-x} \mathrm{Sr}_{x} \mathrm{FeO}_{3}$ from angle resolved photoemission spectra. Phys. Rev. B 74, 115114 (2006).

88. Aizaki, S. et al. Self-energy on the low- to high-energy electronic structure of correlated metal SrVO 3 . Phys. Rev. Lett. 109, 056401 (2012).

89. Yoshimatsu, K. et al. Metallic quantum well states in artificial structures of strongly correlated oxide. Science 333, 319-322 (2011).
90. Chang, Y. J. et al. Layer-by-layer evolution of a two-dimensional electron gas near an oxide interface. Phys. Rev. Lett. 111, 126401 (2013).

91. Chang, C. C. General formalism for quantitative auger analysis. Surf. Sci. 48, 9-21 (1975).

92. Wang, H. Q. et al. Studies of the electronic structure at the $\mathrm{Fe}_{3} \mathrm{O}_{4}-\mathrm{NiO}$ interface. J. Vac. Sci. Technol. A 22, 1675-1681 (2004).

93. Wang, H. Q., Altman, E. I. \& Henrich, V. E. Measurement of electronic structure at nanoscale solid-solid interfaces by surface-sensitive electron spectroscopy. Appl. Phys. Lett. 92, 012118 (2008).

94. Wang, H. Q. et al. Determination of electronic structure of oxide-oxide interfaces by photoemission spectroscopy. Adv. Mater. 22, 2950-2956 (2010).

95. Tanuma, S., Powell, C. J. \& Penn, D. R. Calculations of electron inelastic mean free paths (IMFPS). IV. Evaluation of calculated IMFPs and of the predictive IMFP formula TPP-2 for electron energies between 50 and $2000 \mathrm{eV}$. Surf. Interface Anal. 20, 77-89 (1993).

96. Tanuma, S., Powell, C. J. \& Penn, D. R. Calculations of Electron Inelastic Mean Free Paths (IMFPS) VI. AnalYsis Of The Gries Inelastic Scattering Model And Predictive IMFP equation. Surf. Interface Anal. 25, 25-35 (1997).

97. Shah, A. B. et al. Probing interfacial electronic structures in atomic layer $\mathrm{LaMnO}_{3}$ and $\mathrm{SrTiO}_{3}$ superlattices. Adv. Mater. 22, 1156-1160 (2010).

98. van der Zaag, P. J. et al. On the construction of an $\mathrm{Fe}_{3} \mathrm{O}_{4}$-based all-oxide spin valve. J. Magn. Magn. Mater. 211, 301-308 (2000).

99. van der Heijden, P. A. A. et al. Evidence for roughness driven $90^{\circ}$ coupling in $\mathrm{Fe}_{3} \mathrm{O}_{4} / \mathrm{NiO} / \mathrm{Fe}_{3} \mathrm{O}_{4}$ trilayers. Phys. Rev. Lett. 82, 1020-1023 (1999).

100. Borchers, J. A. et al. Polarized neutron diffraction studies of exchange-coupled $\mathrm{Fe}_{3} \mathrm{O}_{4} / \mathrm{NiO}$ superlattices. J. Appl. Phys. 85, 5883-5885 (1999).

101. Borchers, J. A. et al. Detection of field-dependent antiferromagnetic domains in exchange-biased $\mathrm{Fe}_{3} \mathrm{O}_{4} / \mathrm{NiO}$ superlattices. Appl. Phys. Lett. 77, 4187-4189 (2000).

102. Terashima, T. \& Bando, Y. Formation and magnetic properties of artificial superlattice of $\mathrm{CoO}-\mathrm{Fe}_{3} \mathrm{O}_{4}$. Thin Solid Films 152, 455-463 (1987).

103. Fork, D. K., Philips, J. M., Ramesh, R., Wolf, R. M. Epitaxial oxide thin films and heterostructures. In Mater. Res. Soc. Symp. Proc (ed. Pittsburgh, P. A.) 341, 23-28 (1994).

104. Fadley, C. S. et al. Photoelectron diffraction: new dimensions in space, time, and spin. J. Electron Spectrosc. Relat. Phenom. 75, 273-297 (1995).

105. Zheng, J. C. et al. Simulations of X-ray photoelectron diffraction experiment from theoretical calculations. Surf. Rev. Lett. 8, 549-557 (2001).

106. Zheng, J. C. et al. Atomic-scale structure of the fivefold surface of an AlPdMn quasicrystal: a quantitative $\mathrm{x}$-ray photoelectron diffraction analysis. Phys. Rev. $B$ 69, 134107 (2004).

107. Zheng, J. C. et al. On the sensitivity of electron and X-ray scattering factors to valence charge distribution. J. Appl. Crystallogr. 38, 648-656 (2005).

108. Zheng, J. C., Wu, L. J. \& Zhu, Y. M. Aspherical electron scattering factors and their parameterizations for elements from $\mathrm{H}$ to Xe. J. Appl. Crystallogr. 42, 1043-1053 (2009).

109. Zheng, J. C. et al. Nanoscale disorder and local electronic properties of $\mathrm{CaCu}_{3} \mathrm{Ti}_{4} \mathrm{O}_{12}$ : an integrated study of electron, neutron, and $\mathrm{x}$-ray diffraction, $\mathrm{x}$ ray absorption fine structure, and first-principles calculations. Phys. Rev. B 81, 144203 (2010).

110. Zheng, J. C. \& Wang, H. Q. Principles and applications of a comprehensive characterization method combining synchrotron radiation technology, transmission electron microscopy, and density functional theory. Sci. Sin.: Phys., Mech. Astronom. 51, 030007 (2021). 